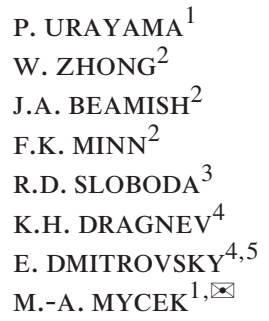

\title{
A UV-Visible-NIR fluorescence lifetime imaging microscope for laser-based biological sensing with picosecond resolution
}

\author{
${ }^{1}$ Dept. of Biomedical Engineering, University of Michigan, Ann Arbor, MI 48109, USA \\ ${ }^{2}$ Dept. of Physics and Astronomy, Dartmouth College, Hanover, NH 03755, USA \\ ${ }^{3}$ Dept. of Biological Science, Dartmouth College, Hanover, NH 03755, USA \\ ${ }^{4}$ Dept. of Medicine, Dartmouth Medical School, Hanover, NH 03755, USA \\ ${ }^{5}$ Dept. of Pharmacology and Toxicology, Dartmouth Medical School, Hanover, NH 03755, USA
}

\begin{abstract}
Received: 23 February 2003
Published online: 22 May 2003 • (c) Springer-Verlag 2003

ABSTRACT This article describes the design and characterization of a wide-field, time-domain fluorescence lifetime imaging microscopy (FLIM) system developed for picosecond time-resolved biological imaging. The system consists of a nitrogen-pumped dye laser for UV-visible-NIR excitation (337.1-960 nm), an epi-illuminated microscope with UV compatible optics, and a time-gated intensified CCD camera with an adjustable gate width $\left(200 \mathrm{ps}-10^{-3} \mathrm{~s}\right)$ for temporally resolved, single-photon detection of fluorescence decays with 9.6-bit intensity resolution and $1.4-\mu \mathrm{m}$ spatial resolution. Intensity measurements used for fluorescence decay calculations are reproducible to within $2 \%$, achieved by synchronizing the ICCD gate delay to the excitation laser pulse via a constant fraction optical discriminator and picosecond delay card. A selfconsistent FLIM system response model is presented, allowing for fluorescence lifetimes $(0.6 \mathrm{~ns})$ significantly smaller than the FLIM system response (1.14 ns) to be determined to $3 \%$ of independently determined values. The FLIM system was able to discriminate fluorescence lifetime differences of at least $50 \mathrm{ps}$. The spectral tunability and large temporal dynamic range of the system are demonstrated by imaging in living human cells: UV-excited endogenous fluorescence from metabolic cofactors (lifetime $\sim 1.4 \mathrm{~ns}$ ); and 460-nm excited fluorescence from an exogenous oxygen-quenched ruthenium dye (lifetime $\sim 400 \mathrm{~ns}$ ).
\end{abstract}

PACS 87.64.-t; 87.57.-s

\section{$1 \quad$ Introduction}

Methods of steady-state fluorescence intensity microscopy are widely used in biology and medicine to reveal information on anatomical features, cellular morphology, cellular function, and intracellular microenvironments, such as ion concentrations, protein binding, lipid content, and membrane status [1,2]. Measurements of fluorophore excited-state lifetimes offer an additional source for contrast for imaging applications, while being generally independent of factors

Fax:+1-734/9361-905, E-mail: mycek@umich.edu influencing steady-state measurements such as fluorophore concentration, photobleaching, and artifacts arising from optical loss, including absorption and scattering $[3,4]$. Therefore, lifetimes reveal non-radiative decay phenomena in turbid cellular environments that might be difficult to quantify via steady-state intensity measurements of quantum yield.

First reported in 1989 [5], fluorescence lifetime imaging microscopy (FLIM) has been used to probe fluorophore micro-environments, including $\mathrm{pH}$, dissolved gas concentration, molecular associations, and energy transfers (for reviews, see [4,6-10]). For example, Sanders et al. [11] reported using FLIM for quantitative $\mathrm{pH}$ determination in living cells. The fluorescent probe c.SNAFL-1 was used to contrast the traditional ratiometric technique with lifetime imaging. In the FLIM method, the lifetime of the protonated and ionized forms of the probe differed, thus revealing the intracellular $\mathrm{pH}$. The study found that both methods provided accurate information regarding intracellular $\mathrm{pH}$, while the lifetime method was easier to employ because no lengthy system calibration was required. FLIM has been used to measure dissolved oxygen concentration in single living cells. Gerritsen et al. [12] used J774 macrophages, incubated with the fluorescent probe ruthenium tris $\left(2,2^{\prime}\right.$-dipyridyl) dichloride hydrate prior to FLIM measurements. Because the probe's fluorescence emission was dynamically quenched by oxygen, the probe's lifetime was directly dependent upon local oxygen concentration and could be used for quantitative imaging in cells via FLIM. Molecular associations, such as binding of the endogenous fluorophore nicotinamide adenine dinucleotide (NADH) to malate dehydrogenase protein, have also been imaged with FLIM [13] by using the increase in NADH lifetime upon binding as a source of contrast. Finally, Oida et al. [14] monitored endosomal fusion in single living cells by imaging fluorescence resonance energy transfer (FRET) between the phospholipids NBD-PE (energy donor) and LRB-PE (energy acceptor). FRET involves a non-radiative transfer of energy between fluorophores, occurring when an energetically excited donor molecule dipole couples to an acceptor molecule. Conditions affecting FRET efficiency include proximity of the donor-acceptor pair (typically $1-10 \mathrm{~nm}$ ), relative orientation, and spectral overlap between the donor's emission band and the acceptor's excitation band [3]. FRET can be 
observed by monitoring either the increase (decrease) in acceptor (donor) fluorescence emission intensity or the increase (decrease) in acceptor (donor) lifetime. Given the advantages of fluorescence lifetime methods mentioned above, FLIM offers an attractive means of performing quantitative FRET experiments with high spatial-sensitivity for sub-cellular localization and binding studies in living cells, where intensity artifacts may complicate the interpretation of steady-state fluorescence intensity measurements.

An important application of FLIM is the imaging of endogenous fluorescence in cells and tissues. While exogenous fluorophores provide useful and specific tools for enhancing contrast, endogenous fluorophores - fluorescent biomolecules indigenous to biological cells and tissues are of biomedical interest as potential probes of metabolic function, tissue morphology, and as intrinsic biomarkers of disease. Because exogenous agents are not employed, endogenous fluorescence methods raise no concerns regarding contrast agent toxicity, delivery, or uptake. Endogenous fluorescence has potential diagnostic importance in medicine, for example in the early detection of cancers (reviewed in [15]), with tissue fluorescence lifetime being a potential source for contrast. Many endogenous fluorophores associated with metabolic activity and tissue morphology have excitation spectra in the near-UV and visible with lifetimes in the subnanosecond to $10 \mathrm{~ns}$ regime, thus motivating the development of picosecond resolution FLIM systems capable of exciting fluorescence at such wavelengths.

Near-UV FLIM is often performed in a raster-scanning mode using two-photon excitation [16, 17]. A mode-locked Ti:Sapphire laser is a common excitation source, providing a typical spectral range of 700-1000 nm for two-photon excitation (corresponding to a one-photon excitation range of $350-500 \mathrm{~nm}$ ) [18]. Here we report a wide-field FLIM system capable of near-UV excitation using a pulsed nitrogenpumped dye laser. The system has a broad excitation range from near-UV to NIR wavelengths (337.1-960 nm), thereby providing access to excitation wavelengths used most often in biological fluorescence microscopy. Direct UV excitation of endogenous fluorophores with a nitrogen laser source provides a less expensive and potentially portable alternative to multi-photon excitation. A nitrogen source emits at $337.1 \mathrm{~nm}$, a wavelength commonly used in clinical endogenous fluorescence studies [15]. Non-imaging, time resolved clinical spectrofluorimeters based on nitrogen lasers have been reported [19-21], thereby making comparisons between in vitro imaging and in vivo non-imaging studies possible.

Fluorophore lifetimes can be measured in either the time domain, in which the system's impulse response to a pulsed excitation is probed, or the frequency domain, in which the system's harmonic response to a modulated excitation is measured [3]. This paper presents a wide-field, timedomain FLIM system, designed for fluorescence lifetime imaging using UV-visible-NIR excitation. Section 2 describes the instrumentation, including the optics, timing and delay electronics, and the gated intensified charge-coupled device (ICCD) system. Section 3 addresses data acquisition issues, including a description of the user interface, system timing, ICCD gating, and system responses. System characterization, calibration, data analysis, and evaluation of measured life- times are treated in Sect. 4. Applications of lifetime imaging in living human cells are described in Sect. 5, followed by a general discussion in Sect. 6.

\section{Instrumentation}

A wide-field microscopy system was developed to obtain two-dimensional fluorescence lifetime images of biological samples with picosecond resolution. A schematic of the FLIM system is shown in Fig. 1a. An excitation pulse $E(t)$ illuminated the sample and wide-field images of fluorescence emission were taken by the ICCD at a controllable intensifier gate delay $t_{\mathrm{G}}$, with intensities integrated over the gate width $\Delta t$. Fluorescence lifetime images were determined by obtaining fluorescence intensity images at several gate delays and
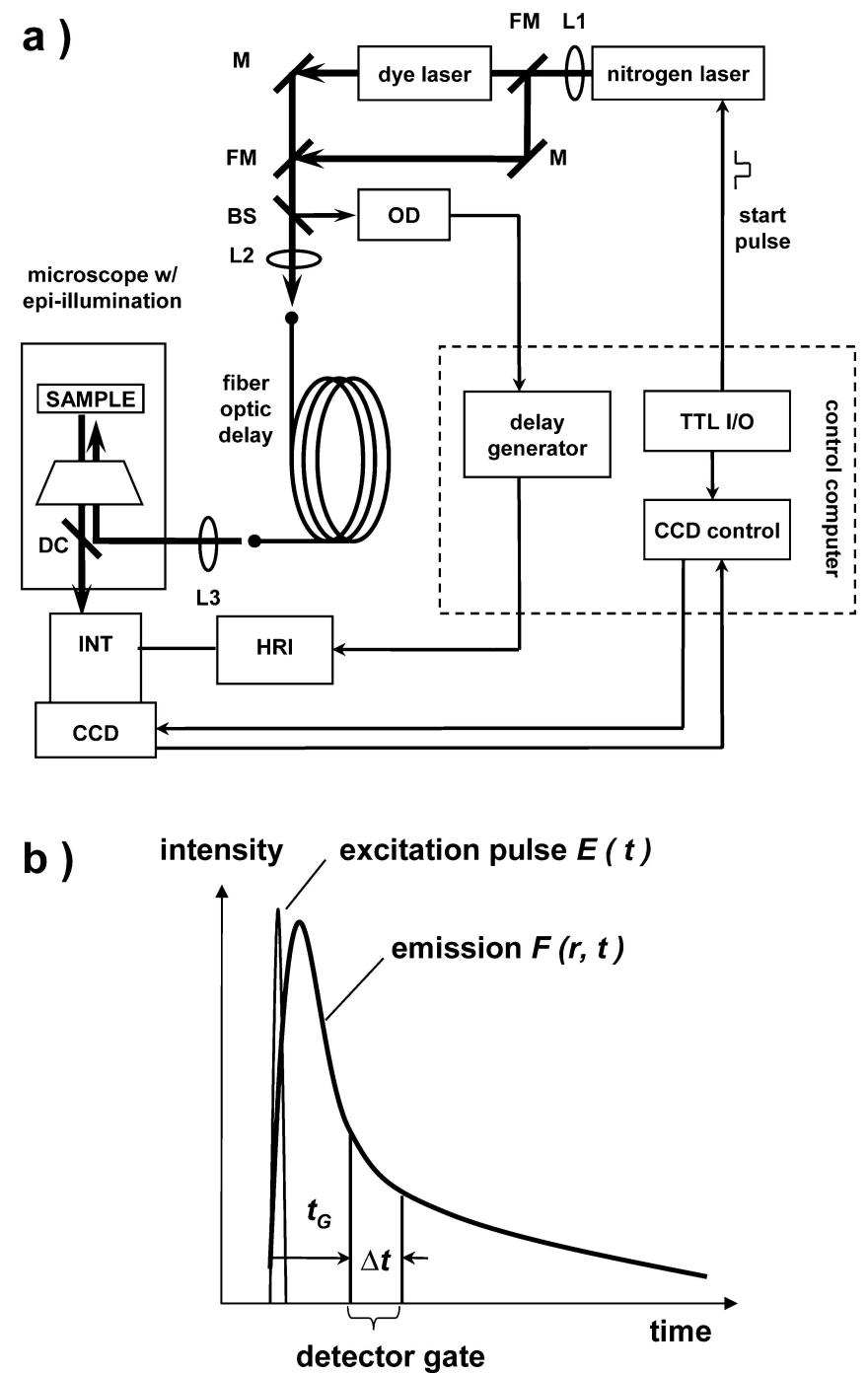

FIGURE 1 a FLIM setup. Abbreviations: CCD, charge-coupled device; HRI, high rate imager; INT, intensifier; TTL I/O, TTL input/output card; OD, optical discriminator; BS, beam splitter; DC, dichroic mirror; FM, "flippable" mirror; L1, L2, L3, quartz lenses; M, mirror. Thick solid lines = light path; thin solid line = electronic path. b FLIM concept. The system is designed to capture the fluorescence emission image at a time $t_{\mathrm{G}}$ after the excitation pulse. The gated ICCD integrates the decay during the interval $\Delta t$. A fluorescence lifetime image is calculated using time-resolved images captured at several $t_{\mathrm{G}}$ 
analyzing the fluorescence intensity decay on a pixel-by-pixel basis. Figure $1 \mathrm{~b}$ shows the concept behind time-domain lifetime determination, with detailed analysis methods presented in Sect. 4. The FLIM instrumentation consisted of (1) optical components for sample excitation, (2) electronics for gate delay control, and (3) a gated ICCD for image detection.

\section{1}

\section{Optical components}

The excitation source was a pulsed nitrogen laser (GL-3300, Photon Technology International, Lawrenceville, $\mathrm{NJ})$ emitting at $337.1 \mathrm{~nm}$ with a spectral bandwidth of $0.1 \mathrm{~nm}$. The laser was capable of operating at repetition rates between 1 and $20 \mathrm{~Hz}$ and had an output energy of $1.45 \mathrm{~mJ}$ per pulse with a full-width-at-half-maximum (FWHM) pulse duration of $0.6 \mathrm{~ns}$. The output of the nitrogen laser was used either for sample excitation or for pumping a tunable, pulsed dye laser (GL-301, Photon Technology International) with a wavelength range of $365-960 \mathrm{~nm}$, depending on the dye used. In order to allow easy access to the nitrogen laser output for UV excitation, the dye laser was not placed flush with the face of the pump laser. Instead, a long focal length quartz lens ( $f=450 \mathrm{~mm}$; L1, Fig. 1a) was used to collimate the nitrogen laser output for optimal dye laser pumping. Light paths could be easily switched for UV or visible excitation by means of mirrors housed on hinged Flipper mounts (9891, New Focus, San Jose, CA; FM, Fig. 1a), which can "flip" mirrors at right angles out of the beam path. Dielectric mirrors with high UV reflectance (5100, New Focus; or BB1-E01, Thorlabs Inc., Newton, NJ) were used in the Flipper mounts to minimize loss as the beam was redirected.

The selected excitation light was coupled into a $20-\mathrm{m}$ long 600- $\mu$ m-diameter 0.22-NA optical fiber (SFS600/660 N, Fiberguide Industries, Stirling, NJ) by means of a quartz lens ( $f=35 \mathrm{~mm}$; L2, Fig. 1a) and delivered to a research grade, inverted microscope (Axiovert S100 2TV with lateral and base TV camera ports, Zeiss, Germany) operating in epiillumination mode. The excitation light exiting the fiber was collimated using a quartz lens ( $f=70 \mathrm{~mm}$; L3, Fig. 1a) and entered the microscope via the back port of the microscope designed for a commercial UV lamp source. A dichroic mirror (350LPDC for 337.1-nm excitation or Q495LP for 460-nm excitation, Chroma Technology Corp., Brattleboro, VT) reflected the beam into a UV compatible Fluar $(40 \times, 1.3$ NA, Zeiss) objective for sample excitation. The entrance port was overfilled, and the objective field of view was centered on the peak of the beam profile. An iris before the dichroic was used to control the size of illumination area, providing uniform illumination with a typical diameter of $150 \mu \mathrm{m}$. Energies at the sample were up to $60 \mu \mathrm{J} /$ pulse at $337.1 \mathrm{~nm}$ and $20 \mu \mathrm{J} /$ pulse at $460 \mathrm{~nm}$ excitation with the entrance iris full-open. To reduce contamination from background fluorescence, the iris was closed until the signal-to-background level was optimized. Due to the low coherence of the source, no speckle was observed.

\section{2}

\section{Timing and variable delay electronics}

To minimize timing artifacts due to temporal jitter in the laser discharge, a reference pulse split from the main laser itself using a beam splitter (BS, Fig. 1a) was used as the timing reference. In addition, the system was operated in a mode in which fluorescence images were acquired from emission due to the same pulse that triggered the timing, rather than synchronized to a later pulse, a common method for time-domain FLIM systems using high repetitionrate mode-locked sources in which there is low pulse-to-pulse jitter.

An electronic pulse with high temporal stability with respect to the reference beam was generated by means of a constant fraction optical discriminator (OCF-400, Becker \& Hickl GmbH, Berlin, Germany). Due to constant fraction discrimination, the optical discriminator reference had a time walk of $<30 \mathrm{ps} \mathrm{rms}$ for a source with $10 \%$ amplitude fluctuation. Output from the optical discriminator was sent to a picosecond delay generator (DEL450, Becker \& Hickl GmbH), which provided an adjustable time delay with 12-bit delay resolution and a minimum jitter of $10 \mathrm{ps}$ or $0.05 \%$ of the full delay range. The delay generator output was used to trigger the gated ICCD camera.

\subsection{Intensified CCD camera}

Time-domain FLIM systems often use gated imaging detectors, typically employing a gated image intensifier coupled to a CCD camera. The intensifier gate, which acts as a camera shutter, is typically in the nanosecond range [22, 23]. An important recent development in ICCD technology is the reduction of the gate width into the $10^{1}-10^{2} \mathrm{ps}$ regime $[24,25]$. Advances in ICCD technology have made near-UV sources, like pulsed nitrogen-pumped dye lasers, an attractive light source for time-domain FLIM of endogenous biological fluorescence. These high flux ( $1.45 \mathrm{~mJ} /$ pulse $)$ laser sources utilize the ICCD's ultra-fast gated imaging ability to obtain rapid, time-resolved, wide-field images, thereby eliminating the need for raster scanning.

After sample excitation, the fluorescence emission was collected through the microscope objective and sent to the lateral TV port of the microscope. A base port in which signal exits the microscope directly without any beam deflection was available for weakly emitting samples, though the port was not needed for this study. Images were collected through a band-pass filter appropriate to the emission spectrum of the fluorophore placed directly in front of the ICCD.

An ultra-fast gated ICCD camera (Picostar HR, La Vision, Goettingen, Germany) was used for image detection. The intensifier used a voltage-gated microchannel plate (MCP) controlled via a high rate imager (HRI, Kentech Instruments, Oxfordshire, UK) for single-photon detection sensitivity. Gating of the intensifier was achieved by applying a pulsed negative voltage across a photocathode in front of the MCP. The gate width was controlled using an ultrashort pulse generated by the HRI, with nominal gate width settings from 200 to $1000 \mathrm{ps}$. Longer gate widths were produced by slaving the ICCD gate to an external logic signal for gate widths adjustable from $10^{-9}$ to $10^{-3} \mathrm{~s}$. A voltage across the MCP, adjustable from 260 to $800 \mathrm{~V}$, provided a variable intensifier gain. The output of the intensifier was coupled to a $640 \times 480$ pixel, 12-bit CCD camera, cooled to $-15^{\circ} \mathrm{C}$ to minimize thermal dark current. The electronic readout noise was less than 2 counts over the en- 


\begin{tabular}{|c|c|c|}
\hline Component & Parameter & Value \\
\hline \multicolumn{3}{|l|}{ intensifier } \\
\hline & spectral range $(>4 \% \mathrm{QE})$ & $300-650 \mathrm{~nm}$ \\
\hline & gate widths & $200 \mathrm{ps}-10^{-3} \mathrm{~s}$ \\
\hline & microchannel plate voltage & $260-800 \mathrm{~V}$ \\
\hline & resolution & $35 \mathrm{lp} / \mathrm{mm}$ \\
\hline \multicolumn{3}{|l|}{ CCD } \\
\hline & spectral range $(>4 \% \mathrm{QE})$ & $300-880 \mathrm{~nm}$ \\
\hline & number of pixels & $640 \times 480$ \\
\hline & pixel size & $9.9 \mu \mathrm{m} \times 9.9 \mu \mathrm{m}$ \\
\hline & dynamic range & 12 bit \\
\hline & readout rate & $12.5 \mathrm{MHz}$ \\
\hline & readout noise & 2 counts@12.5 MHz \\
\hline & frame rate & 30 frames $/ \mathrm{s}$ \\
\hline & exposure times & $1 \mathrm{~ms}-1000 \mathrm{~s}$ \\
\hline & full well capacity & 35000 electrons \\
\hline & dark current & $<0.1$ electrons $/$ pixel $/ \mathrm{s}$ \\
\hline & A/D conversion factor & 7.5 electrons/count \\
\hline \multicolumn{3}{|c|}{ optical discriminator } \\
\hline & spectral range & $300-1000 \mathrm{~nm}$ \\
\hline & time walk ( $1 \mathrm{~ns}$ input, $10 \%$ stability) & $<30 \mathrm{ps} \mathrm{rms}$ \\
\hline \multicolumn{3}{|l|}{ delay card } \\
\hline & delay range & $10 \mathrm{~ns}-100 \mu \mathrm{s}$ \\
\hline & absolute delay accuracy & $5 \%$ \\
\hline & delay resolution & 12 bit, 2.5 ps minimum \\
\hline & delay jitter & $0.05 \%$ of range, $10 \mathrm{ps}$ minimum \\
\hline \multicolumn{3}{|l|}{ optical fiber } \\
\hline & material & silica \\
\hline & core diameter & $600 \mu \mathrm{m}$ \\
\hline & clad diameter & $660 \mu \mathrm{m}$ \\
\hline & numerical aperture & 0.22 \\
\hline & attenuation factor & $0.25 \mathrm{~dB} / \mathrm{m}$ at $337 \mathrm{~nm}$ \\
\hline & refractive index (at $633 \mathrm{~nm}$ ) & core: 1.457 , clad: 1.44 \\
\hline
\end{tabular}

TABLE 1 Specifications of FLIM components tire image. Table 1 summarizes additional instrumentation parameters.

\section{3}

\section{System parameters}

This section discusses issues related to data acquisition, describing the user interaction with the system during a typical imaging session and the system timing employed to obtain images at controllable delays. Next, we characterize the total response of the system $(1.14 \mathrm{~ns}$ at $337.1 \mathrm{~nm}$, $0.90 \mathrm{~ns}$ at $460 \mathrm{~nm}$ ), including responses of the various components of the FLIM system such as the ICCD gate width $(0.62 \mathrm{~ns}$ at the shortest setting) and laser pulse durations $(0.86 \mathrm{~ns}$ at $337.1 \mathrm{~nm}, 0.41 \mathrm{~ns}$ at $460 \mathrm{~nm})$. We also present a self-consistent model relating these responses (Sects. 3.3 and 3.4).

\subsection{User interface and software}

The setup described in the previous section was designed to image the sample fluorescence at a user settable gate delay $t_{\mathrm{G}}$ (Fig. 1b). The system executed the following sequence per each fluorescence image acquisition (see Fig. 1a for setup): the control computer TTL I/O card sent a trigger signal to fire the nitrogen laser and start the ICCD camera exposure; the laser output was split into an excitation and reference pulse (BS); the reference pulse initiated the user controlled electronic delay via the optical discriminator (OD), while the excitation pulse was delayed through a fiber optic; the excitation pulse entered the microscope and excited the sample, with the ICCD capturing the resulting fluorescence image at the controlled time $t_{\mathrm{G}}$ after excitation. After completion of this sequence, the CCD was read off and the image was sent to the computer for analysis and storage. Images from several delays were used to compute fluorescence lifetime maps, as described in Sect. 4.

The software was designed as both an instrument control interface and a data analysis platform. A commercial software package (DaVis v6, LaVision) contained applications for camera control, computer card control, and image analysis, including a $\mathrm{C}$-like macro programming environment with a graphical user interface (GUI). Images from the ICCD were stored in memory buffers, and could be manipulated mathematically and written to disk. A CCD dark current correction was applied to each fluorescence image, and consisted of subtracting off an image averaged over five ICCD exposures taken without opening the intensifier gate.

A macro and GUI was written for the collection and processing of images for FLIM. The user could input up to 9 ICCD gate delays and acquire images averaged over several pulses at a given delay. A typical session might consist of using 3 gate delays in the calculation of a fluorescence lifetime image with 1 to 5 images averaged per delay. Before calculating a fluorescence lifetime map, a region of interest (ROI) representing the background signal could be set for subtraction from each intensity image. Weak pixels, which contribute noise to the lifetime image, were rejected if below a usersettable threshold. Finally, the average fluorescence lifetime in a ROI in the image could be determined by producing a histogram of lifetimes. 
The CCD exposure time ( $200 \mathrm{~ms}$ ) was controlled independently of the intensifier gating in order to accommodate the large temporal jitter in the TTL I/O card used to trigger the laser and start the exposure of the CCD. The CCD exposure time and the delay between the start of CCD exposure and laser triggering could be adjusted by the user to optimize data acquisition rates.

\subsection{System timing}

Time-domain lifetime determination requires accurate and precise knowledge of the time between sample excitation and signal detection. The ICCD gate was synchronized to an electrical pulse triggered by a reference laser pulse split from the excitation pulse and detected by means of a constant fraction optical discriminator, as shown in Fig. 1a.

Due to inherent delays in the electronics and signal propagation through the electronic cabling, the minimum time from the triggering of the optical discriminator to the opening of the ICCD shutter was $70 \mathrm{~ns}$. Because fluorescence lifetimes of interest are in sub-nanosecond regime, the ICCD shutter must open within hundreds of picoseconds after sample excitation for accurate sampling of the fluorescence decay. Therefore, the excitation pulse was time delayed in order to bring the fluorescence emission into a regime electronically accessible by the picosecond delay card and ICCD gate. An optical delay path was introduced by means of a $\sim 20$-m-long optical fiber, which had the added benefit of homogenizing the spatial intensity distribution of the beam. The measured propagation time through the fiber was $102.5 \mathrm{~ns}$, indicating a fiber length of $21.33 \mathrm{~m}$ with an index of refraction of $n=1.44$. Rather than using a single-mode fiber, multimode distortion within the fiber was minimized while maximizing power throughput by underfilling the fiber.

\subsection{The total system response and ICCD gate widths}

In this system, the ICCD gate effectively operated in a single shot mode. Because the HRI was optimized for high repetition rates of up to $100 \mathrm{MHz}$ for use with mode-locked lasers, the ICCD gate characteristics in low repetition mode were evaluated here. The ICCD gate widths were estimated by comparing the total system response at the sample (the temporal signal profile measured by the ICCD from a sample with negligibly short fluorescence lifetime) with the increase in integrated intensity upon increasing the ICCD gate width at a fixed gate delay.

To measure the total system response, the intensity over a range of delays was measured for rose bengal in de-ionized water. The solution was placed between a quartz slide (01018AB, SPI Supplies, West Chester, PA) and \#1 glass coverslip, held in place using a Press-to-Seal silicone spacer (P-18174, Molecular Probes, Eugene, OR). The fluorescence lifetime of rose bengal was $0.09 \mathrm{~ns}$ [26], shorter than the lower limit of the ICCD gate width $(0.2 \mathrm{~ns}$, as specified by the manufacturer). Figure $2 \mathrm{a}$ shows the temporal profile of rose bengal in de-ionized water imaged using the FLIM system at $337.1 \mathrm{~nm}$ excitation for three nominal gate width settings of 200,500 , and $1000 \mathrm{ps}$. The intensity at a given delay was the average of a 4500 pixel ROI. The entire profile was then normalized to the peak intensity. Measured profiles were fit to Gaussians and

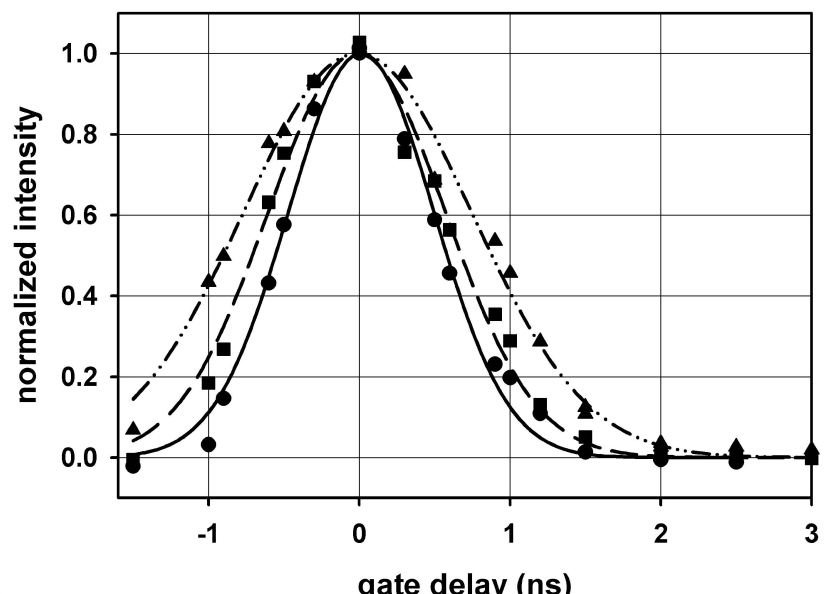

a

gate delay (ns)

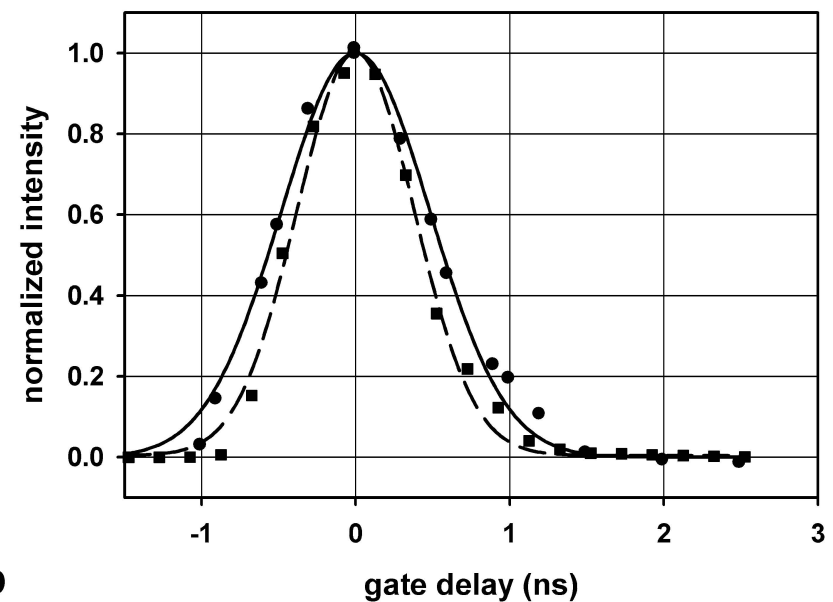

FIGURE 2 FLIM system response determined by measuring the fluorescence intensity of rose bengal in de-ionized water versus the ICCD gate delay. a Response using 337.1-nm excitation at several nominal gate width

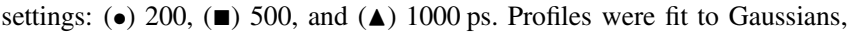
for FWHM of 1.139, 1.387, and 1.783 ns, respectively. b A comparison between system responses using: $(\bullet)$ the nitrogen laser $(337.1 \mathrm{~nm})$, and $(\boldsymbol{\square})$ the dye laser $(460 \mathrm{~nm})$. The nominal gate width was $200 \mathrm{ps}$. Profiles were fit to Gaussians, for FWHM of 1.139 and 0.899 ns, respectively

FWHM values were $1.139,1.387$, and $1.783 \mathrm{~ns}$ for the three nominal gate width settings, respectively.

The data plotted in Fig. 2a represented the total FLIM system response, i.e., the excitation pulse as detected by the ICCD ( $\left.S R_{\text {exICCD }}\right)$, which can be separated into contributions from three sources as follows:

$S R_{\mathrm{exICCD}}^{2}=S R_{\mathrm{gw}}^{2}+S R_{\mathrm{ex}}^{2}+S R_{\mathrm{micr}}^{2}$,

assuming Gaussian responses, with $S R_{\mathrm{gw}}$ the effective ICCD gate width, $S R_{\mathrm{ex}}$ the incident excitation pulse duration, and $S R_{\text {micr }}$ the response of the microscope optics. Note the increase in FWHM with increasing gate width setting, as expected, due to an increasing $S R_{\mathrm{gw}}$.

Next, $S R_{\text {exICCD }}$ was compared with the signal intensity for a constant intensity input, which should be proportional to the integration time (that is, $S R_{\mathrm{gw}}$ ). Because a stable DC source was not available, the intensity of a long lifetime fluorophore was used to give a near constant input and was imaged at a given delay using the same gate width settings as Fig. 2a. 
9-cyanoanthracene in ethanol ( $\tau=14.2 \mathrm{~ns}$ ) [21] was chosen because it had a lifetime an order of magnitude greater than the nominal gate settings. The ratio of $S R_{\mathrm{gw}}$ at two gate width settings, $\mathrm{A}$ and $\mathrm{B}$, should be related to the measured intensities, $I_{\mathrm{A}}$ and $I_{\mathrm{B}}$, by

$\frac{S R_{\mathrm{gw}, \mathrm{A}}^{2}}{S R_{\mathrm{gw}, \mathrm{B}}^{2}}=\frac{S R_{\mathrm{exICCD}, \mathrm{A}}^{2}-S R_{\mathrm{ex}, \mathrm{A}}^{2}}{S R_{\mathrm{exICCD}, \mathrm{B}}^{2}-S R_{\mathrm{ex}, \mathrm{B}}^{2}}=\frac{\left(I_{\mathrm{A}}\right)^{2}}{\left(I_{\mathrm{B}}\right)^{2}}$,

assuming $S R_{\text {micr }}$ is small. We solved for $S R_{\mathrm{ex}}$, which was assumed identical for settings A and B ( $S R_{\mathrm{ex}}$ was a property of the laser), by substituting measured values for $S R_{\text {exICCD }}$ and $I$, obtaining a value of $S R_{\mathrm{ex}}=0.957 \pm 0.117 \mathrm{~ns}$, the effective laser pulse duration at the sample. Thus, knowing $S R_{\mathrm{ex}}$, we obtained $S R_{\mathrm{gw}}=0.617,1.004$, and $1.504 \mathrm{~ns}$ for the short, intermediate, and long gate width settings, respectively.

\subsection{Laser pulse durations and other system responses}

The system response of various components of the FLIM system was modeled to more completely understand its relation to the temporal resolution of the system. This included characterizing the laser pulse duration before the optical fiber, the multimode distortion in the optical fiber, and the laser pulse duration after the fiber (that is, at the sample). System responses were modeled as Gaussians so that responses added in quadrature. An internally consistent model of the system responses was developed using values obtained from the nitrogen laser output and further validated using values obtained from the 460-nm dye laser output.

The laser pulse duration at the sample, $S R_{\mathrm{ex}}$, estimated in the previous section $(0.957 \mathrm{~ns})$ was confirmed by comparing it to pulse durations measured using an avalanche photodiode (APD) (C5658, Hamamatsu, Japan). We modeled the relationship between the APD and ICCD as

$S R_{\mathrm{exAPD}}^{2}=S R_{\mathrm{ex}}^{2}+S R_{\mathrm{APD}}^{2}$

$S R_{\mathrm{exAPD}}^{2}=S R_{\mathrm{exICCD}}^{2}+S R_{\mathrm{APD}-\mathrm{ICCD}}^{2}$,

where $S R_{\text {exAPD }}$ is the APD measured excitation pulse duration, $S R_{\mathrm{APD}}$ is the instrument response of the APD, and $S R_{\mathrm{APD}-\mathrm{ICCD}}$ is the conversion between APD and ICCD measured laser pulse durations at the sample for a particular ICCD gate width. Combining with (1), we have

$S R_{\mathrm{APD}}^{2}=S R_{\mathrm{gw}}^{2}+S R_{\mathrm{APD}-\mathrm{ICCD}}^{2}$,

The relationships between the system responses can be represented as in Fig. 3, in which the conversion factor is added in quadrature in the direction of the arrow.

The measured FWHM of the UV laser pulse at the sample was $S R_{\mathrm{exAPD}}=1.428 \mathrm{~ns}$. Thus, $S R_{\mathrm{APD}-\mathrm{ICCD}}=0.861$, 0.340 , and $1.068 \mathrm{ins}$, for the short, intermediate, and long gate width settings. Using (4), $S R_{\mathrm{APD}}$ showed good agreement for the three ICCD gate widths with $S R_{\mathrm{APD}}=1.059 \pm$ $0.001 \mathrm{~ns}$, where the error was the standard deviation of three $S R_{\mathrm{APD}}$ estimates. Using the $S R_{\mathrm{APD}}$, the $S R_{\mathrm{ex}}$ was $\sqrt{S R_{\mathrm{exAPD}}^{2}-S R_{\mathrm{APD}}^{2}}=\sqrt{(1.428)^{2}-(1.059)^{2}}=0.958 \mathrm{~ns}$, in agreement with the value previous estimate of $0.957 \pm$ $0.117 \mathrm{~ns}$.

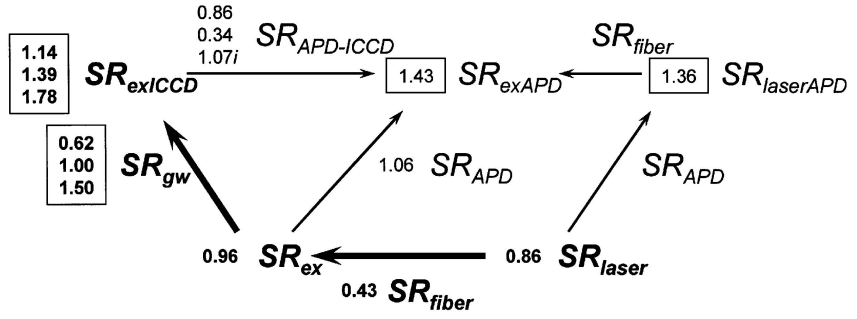

FIGURE 3 System response model. System response values are in units of ns. Components of the FLIM system are linked by bold arrows. Additional parameters not part of the FLIM system but used in the measurement of system responses are linked by thin arrows. Values shown are those using the nitrogen laser (337.1-nm wavelength). System responses with multiple values correspond to an increasing ICCD gate width (top to bottom, 200, 500, and 1000 ps nominal settings). Boxed values are measured responses; see text for experimental details. Responses were assumed to be Gaussian and are added in quadrature in the direction of the arrow. Calculations in the text use three decimal digits to minimize round-off errors. Abbreviations: ex, excitation pulse at the sample after the fiber optic delay; laser, laser pulse before the fiber optic delay; ICCD, measured by ICCD; $A P D$, measured using an APD; $g w$, ICCD gate width; $A P D-I C C D$, the effective conversion between the responses of the APD and ICCD; fiber, multimode distortion through delay fiber

Next, the multimode distortion in the fiber was determined by comparing the APD-measured laser pulse duration after the optical delay fiber, $S R_{\text {exAPD }}$, with the APD-measured laser pulse duration before the optical delay fiber, directly out of the laser, $S R_{\text {laserAPD. Modeling the multimode distortion as }}$

$S R_{\text {fiber }}^{2}=S R_{\text {exAPD }}^{2}-S R_{\text {laserAPD }}^{2}$,

and by assuming $S R_{\mathrm{APD}}$ was independent of APD position, i.e., a true instrument dependent response, we also have

$S R_{\text {fiber }}^{2}=S R_{\mathrm{ex}}^{2}-S R_{\text {laser }}^{2}$,

where $S R_{\text {laser }}$ is the pulse duration of the laser. For the UV laser pulse, $S R_{\text {laserAPD }}$ was $1.362 \mathrm{~ns}$, and therefore, $S R_{\text {fiber }}=$ $0.429 \mathrm{~ns}$, and using (6) gives $S R_{\text {laser }}=\sqrt{(0.958)^{2}-(0.429)^{2}}=$ $0.857 \mathrm{~ns}$. Alternatively, a consistent value is gotten by $S R_{\text {laser }}=\sqrt{S R_{\text {laserAPD }}^{2}-S R_{\mathrm{APD}}^{2}}=\sqrt{(1.362)^{2}-(1.059)^{2}}=$ $0.856 \mathrm{~ns}$. Both are slightly longer than the manufacturerspecified pulse duration of $0.6 \mathrm{~ns}$. Thus, Fig. 3 represents an internally consistent set of relationships between the various system responses in the system.

Gate width and system responses were further validated using the nitrogen laser-pumped dye laser at $460 \mathrm{~nm}$. $S R_{\text {exICCD }}$ for the dye laser (460-nm wavelength) was $0.899 \mathrm{~ns}$ at the nominal 200-ps gate width setting (Fig. 2b). The APD measurements were $S R_{\mathrm{exAPD}}=1.231 \mathrm{~ns}$ and $S R_{\text {laserAPD }}=$ $1.130 \mathrm{~ns}$. Using the previously determined value of $S R_{\mathrm{APD}-\mathrm{ICCD}}$ for the shortest gate width setting $(0.861 \mathrm{~ns})$, we have

$$
\begin{aligned}
S R_{\mathrm{exAPD}}^{2} & =S R_{\mathrm{exICCD}}^{2}+S R_{\mathrm{APD}-\mathrm{ICCD}}^{2} \\
& =(0.899)^{2}+(0.861)^{2}=(1.245)^{2},
\end{aligned}
$$

within $1.1 \%$ of the measured value of $1.231 \mathrm{~ns}$. The laser pulse duration at the sample $S R_{\mathrm{ex}}$, calculated in two ways, also gives a consistent value when using previously estimated values of 


$$
\begin{aligned}
& S R_{\mathrm{gw}} \text { and } S R_{\mathrm{APD}}: \\
& S R_{\mathrm{ex}}^{2}=S R_{\mathrm{exICCD}}^{2}-S R_{\mathrm{gw}}^{2} \\
& =(0.899)^{2}-(0.617)^{2}=(0.654)^{2} \\
& S R_{\mathrm{ex}}^{2}=S R_{\mathrm{exAPD}}^{2}-S R_{\mathrm{APD}}^{2} \\
& =(1.231)^{2}-(1.059)^{2}=(0.628)^{2} .
\end{aligned}
$$

The multimode distortion was

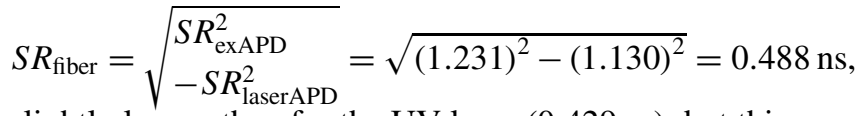

slightly longer than for the UV laser (0.429 ns), but this may be due to variations in the way the pulse was launched into the fiber. Finally, the dye laser pulse duration $S R_{\text {laser }}$ was

$$
\begin{aligned}
S R_{\text {laser }}^{2} & =S R_{\text {laserAPD }}^{2}-S R_{\mathrm{APD}}^{2} \\
& =(1.130)^{2}-(1.059)^{2}=(0.394)^{2} \\
S R_{\text {laser }}^{2} & =S R_{\mathrm{ex}}^{2}-S R_{\text {fiber }}^{2} \\
& =(0.641)^{2}-(0.488)^{2}=(0.416)^{2} .
\end{aligned}
$$

Here we used $S R_{\mathrm{ex}}=0.641 \mathrm{~ns}$, the average of the values in (8). The average value of the laser pulse duration for the dye laser was $S R_{\text {laser }}=0.405 \mathrm{~ns}$, slightly shorter than the manufacturer specified pulse duration of $0.5 \mathrm{~ns}$.

Figure 3 summarizes the system response model presented here, related by nine coupled relationships between 15 variables. Five variables were measured directly, and three were estimated from ratios of fluorescence intensities, reducing the number of unknown variables to seven, making the system sufficiently determined. Consistency was demonstrated amongst the over determined variables, and further validated using measured values for the nitrogen laser-pumped dye laser.

\section{Characterization and lifetime measurement}

Though fluorescence lifetime is independent of excitation intensity, lifetimes were calculated from a series of fluorescence intensity images, and therefore drift or jitter in laser intensity as well as ICCD non-linearities will affect recovered lifetimes. This section characterizes the stability of intensity measurements and ICCD linearity, and evaluates the FLIM system's ability to accurately measure fluorescence lifetimes. Intensity measurements were stable to within $2 \%$ when images were averaged over several excitation pulses, and the ICCD provided a linear response to the input intensity with a resolution of 9.6 bits. Good agreement was observed between FLIM recovered fluorescence lifetimes and lifetimes recovered using an APD for fluorophores with lifetimes ranging from 0.6 to $14 \mathrm{~ns}$. After correcting for system response, there is an agreement of $3 \%$ for the $0.6 \mathrm{~ns}$ lifetime fluorophore, rose bengal in ethanol, when compared with the APD recovered lifetime.

\subsection{Stability of intensity measurement and ICCD linearity}

Because the nitrogen laser was a gas discharge source, factors such as gas flow and pressure affected pulseto-pulse intensity. In addition, electronic delay and ICCD gate jitters may also affect apparent intensities due to the transient nature of the fluorescence signal. Therefore, the ability to measure intensities reproducibly was evaluated by acquiring repeated images of 1,4-Bis(5-phenyloxazol-2yl)benzene (POPOP) in ethanol. The solution was placed between a quartz slide and \#1 glass coverslip, held in place using a silicone spacer. In order to evaluate stability in the detection of both strong and weak intensities, gate delays were chosen to produce images of approximately 200 and 15 ICCD counts. Image intensities were determined as an average value for pixels in a ROI near the center of the image. Standard deviations in the intensity over 16 images were $4.0 \%$ and $5.4 \%$ for the 200 and 15 count images, respectively. By comparison, the nitrogen laser was specified to have a $2.5 \%$ pulse-to-pulse variation. The standard deviation in the mean intensity measurement was larger than the laser intensity jitter due additional effects such as timing and gating jitter. To minimize fluctuations in the intensity measurement, images were averaged over five laser pulses, reducing the standard deviation in the intensity measurement to $1.4 \%$ and $1.7 \%$ for the 200 and 15 count images, respectively.

Next, we characterized the response of the ICCD to input signal strength. Fluorescein $\left(10^{-3} \mathrm{M}\right)$ in de-ionized water was imaged with the excitation intensity and gate delay kept constant, providing a fixed reference intensity. ICCD input intensity was controlled using a set of quartz neutral density filters (03FSQ011, Melles Griot, Carlsbad, CA) placed in front of the ICCD in place of the band-pass filter. The relative input intensity was calculated with respect to the reference intensity from measured optical density values at $510 \mathrm{~nm}$ (the fluorescein emission peak) provided by the manufacturer. Photobleaching was monitored by collecting images in nonsequential order of filter optical densities. The gate width was set to the 200 ps nominal setting and three images were averaged for each neutral density setting and the mean intensity over a 15000 pixel region near the center of the image was plotted as a function of input intensity (Fig. 4). Deviations

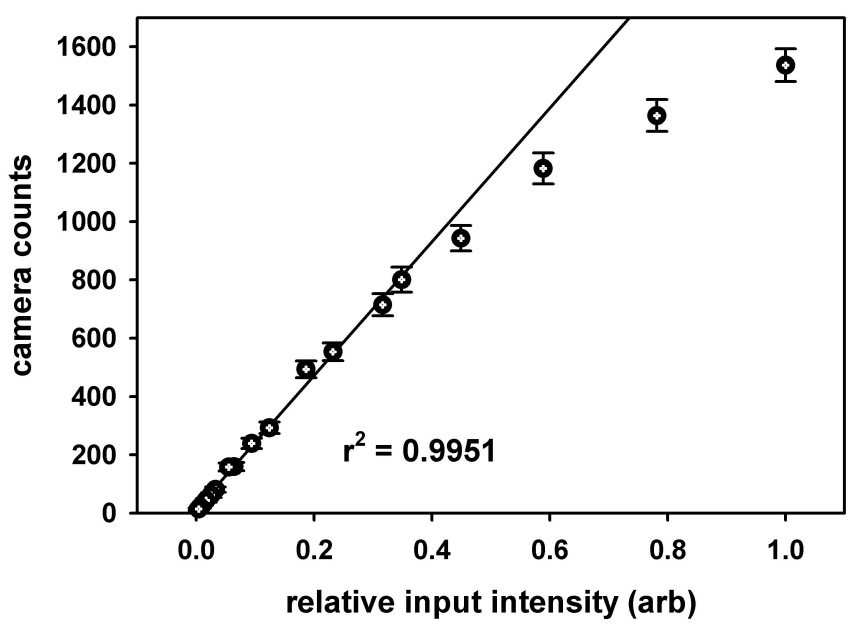

FIGURE 4 ICCD intensity response. The ICCD MCP voltage was $760 \mathrm{~V}$ and the nominal gate width was $200 \mathrm{ps}$. Intensity was determined as an average over a ROI with error bars being the standard deviation. Relative intensities with respect to a reference intensity were varied using a series of neutral density filters. Deviation from linearity was observed above 800 counts 
from linearity were observed above 800 counts, indicating the ICCD intensity resolution was effectively 9.6-bit when operated in the single-shot, ultra-fast gated mode. This does not fully utilize the 12-bit CCD camera capacity, as confirmed by operating the ICCD gate in DC mode and performing the linearity measurement using the microscope lamp as the reference intensity.

\section{2}

\section{Temporal response}

The ability of the system to distinguish fluorophores of different lifetimes was evaluated first by measuring the temporal profile of fluorophores of known lifetimes. Figure 5 shows the intensity decays of fluorophores rose bengal $\left(5 \times 10^{-5} \mathrm{M}\right.$ in ethanol), POPOP (saturated in ethanol), fluorescein $\left(10^{-5} \mathrm{M}\right.$ in de-ionized water), and 9-cyanoanthracene $\left(8 \times 10^{-5} \mathrm{M}\right.$ in ethanol) ranging in lifetimes from 0.6 to $\sim 14 \mathrm{~ns}$. The fluorophores were mounted individually between a slide and coverslip by means of a glass or silicone spacer. Five images were averaged for each gate delay, and the mean intensity over a ROI was plotted as a function of gate delay, normalized to the peak intensity of the profile. Photobleaching was monitored by periodically meas-

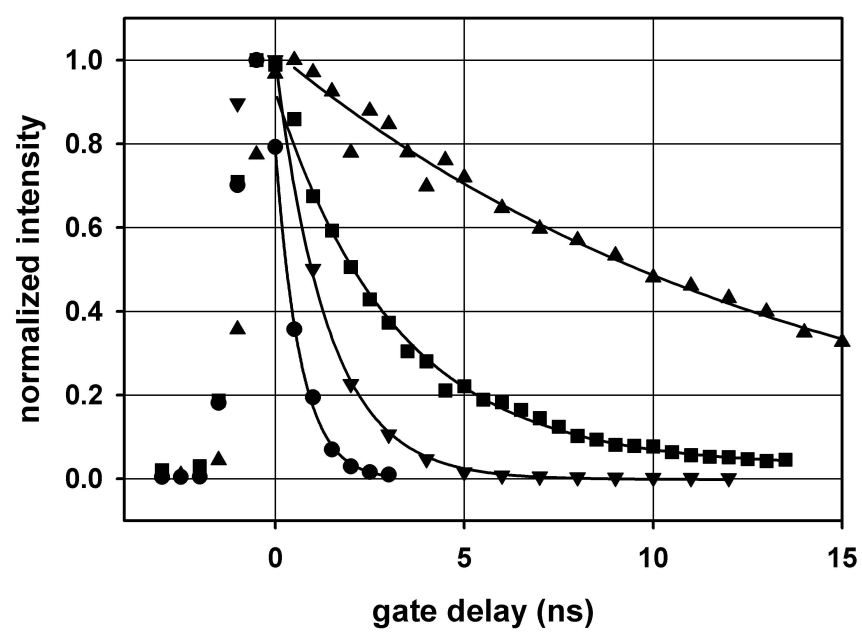

FIGURE 5 FLIM measured fluorescence intensity decays for a range of fluorophore lifetimes: 0.6 to $14 \mathrm{~ns}$. Fluorophore, in order of increasing lifetime:

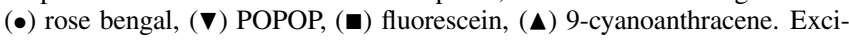
tation was at $337.1 \mathrm{~nm}$. Solid lines are single-exponential fits to the decay. Recovered lifetime values are shown in Table 2 under the "exponential fit" column uring the intensity at a reference delay near the emission profile peak. Decay times were determined from least squares fits to single exponential decays starting at the point $0.5 \mathrm{~ns}$ after the profile peak.

Exponentially fitted lifetimes from the FLIM system showed excellent agreement with lifetimes measured independently using a nitrogen laser-based fluorescence lifetime spectrometer (FLS) described in detail in [21] (Table 2). The non-imaging FLS used an APD module for time-resolved fluorescence detection. The lifetime was determined using an iterative reconvolution algorithm, fit to a single exponential decay, to correct for the FLS system response.

Overall, there was good agreement (Table 2) between the exponentially fit FLIM lifetime compared with the FLS measured lifetime. Importantly, this was true even without the deconvolution of the FLIM system response. This indicated that the effects associated with a nanosecond FLIM system response were not prohibitive in determining sub-nanosecond lifetimes.

\subsection{Lifetime image analysis}

Because fluorescence lifetimes in imaging are determined on a pixel-by-pixel basis, iterative methods for fitting decays can be time consuming. For example, at $1 \mathrm{~ms} /$ pixel, it would take it would take over 5 min to process $640 \times 480$ pixel image. Alternatives are analytic lifetime determination methods such as the two-gate rapid lifetime determination (RLD) algorithm [5, 22, 27]. Assuming a negligibly short excitation pulse, a single exponential fluorescence decay, and an effective gate width $\Delta t$, the gated detector signal $D(r, t)$ at time $t$ is

$D(r, t)=\int_{t}^{t+\Delta t} \alpha \mathrm{e}^{-\left(\frac{t^{\prime}}{\tau(r)}\right)} \mathrm{d} t^{\prime}=\alpha \tau\left[\mathrm{e}^{-\left(\frac{t}{\tau(r)}\right)}-\mathrm{e}^{-\left(\frac{t+\Delta t}{\tau(r)}\right)}\right]$,

where $r$ is the spatial location, often a detector pixel location. A spatially resolved lifetime $\tau(r)$ can be determined by using two images gated at times $t_{1}$ and $t_{2}$, so that

$\tau(r)=\frac{t_{2}-t_{1}}{\ln \left(\frac{D\left(r, t_{1}\right)}{D\left(r, t_{2}\right)}\right)}$

Because a two-gate scheme can be sensitive to noise, we calculated maps of fluorescence lifetimes using an extension of

\begin{tabular}{lccc}
\hline fluorophore & \multicolumn{2}{c}{ FLIM measured } & FLS measured \\
\hline & exponential fit (ns) & 3-gate fit (ns) & deconvolved lifetime (ns) \\
rose bengal $\left(5 \times 10^{-5} \mathrm{M}\right)$ in ethanol & $0.70 \mathrm{~ns}(13 \%)$ & $0.75(21 \%)$ & 0.62 \\
(after FLIM system response correction) & $0.64 \mathrm{~ns}(3.2 \%)$ & & 1.40 \\
POPOP (saturated) in ethanol & $1.38 \mathrm{~ns}(1.4 \%)$ & $1.31(6.4 \%)$ & 3.34 \\
fluorescein $\left(10^{-5} \mathrm{M}\right)$ in de-ionized water & $3.21 \mathrm{~ns}(3.9 \%)$ & $3.25(2.7 \%)$ & 14.2 \\
9-cyanoanthracene $\left(8 \times 10^{-5} \mathrm{M}\right)$ in ethanol & $13.8 \mathrm{~ns}(2.8 \%)$ & $15.6(9.9 \%)$ & \\
\hline
\end{tabular}

TABLE 2 Comparison of fluorescence lifetime measurements. FLIM lifetimes were determined from single exponential fits to the fluorescence decay with intensities integrated over a ROI, and compared to FLIM lifetimes determined using an analytic 3-gate lifetime determination protocol. Both FLIM lifetimes were compared to an independently determined lifetime using a fluorescence lifetime spectrometer (FLS) described in [21]. Percent differences from FLS determined lifetimes are shown in parentheses. Note that the FLIM system response was not deconvolved from FLIM measured lifetimes. Simulations were performed to account for system response effects, resulting in improved lifetime determination for short lifetime fluorophores, as shown for rose bengal 
the RLD method to an arbitrary number of gates [9]. Lifetime maps were calculated on a pixel-by-pixel basis by fitting the lifetime of pixel $p$ to the logarithm of the intensity,

$\ln I_{i, p}=-\frac{t_{i}}{\tau_{p}}+C$

where $I_{i, p}$ is the intensity of pixel $p$ in image $i, t_{i}$ is the gate delay of image $i$, and $C$ is a constant. Analytic least squares lifetime fits were

$\tau_{p}=-\frac{N\left(\sum t_{i}^{2}\right)-\left(\sum t_{i}\right)^{2}}{N \sum t_{i} \ln I_{i, p}-\left(\sum t_{i}\right)\left(\sum \ln I_{i, p}\right)}$,

where $N$ is the number of images. All sums are over $i$.

To evaluate spatial variations in the goodness of the fit of $\tau_{p}$ to (12), the correlation coefficient $r$ was also calculated. The correlation coefficient is a measure of how well a series of points fit a line, with a value of \pm 1 representing a perfect linear correlation. To give only positive values, we map $r^{2}$ using:

$r_{p}^{2}=\frac{\left[N \sum t_{i} \ln I_{i, p}-\left(\sum t_{i}\right)\left(\sum \ln I_{i, p}\right)\right]^{2}}{\left[N \sum t_{i}^{2}-\left(\sum t_{i}\right)^{2}\right]\left[N \sum\left(\ln I_{i, p}\right)^{2}-\left(\sum \ln I_{i, p}\right)^{2}\right]}$.

Calculations were done by creating intermediate images for sums with subscripts $p$. Calculations for creating $640 \times 480$ pixel maps of both lifetime and $r^{2}$ using 3 gates required $10 \mathrm{~s}$ using a PC with an AMD K6-2 processor at $300 \mathrm{MHz}$.

With this, we evaluated the ability of the multi-gate lifetime protocol to determine and discriminate lifetimes. Samples were prepared by loading the fluorescent dyes into quartz capillaries (50- $\mu \mathrm{m}$ inner diameter; CV0508Q, VitroCom Inc., Mountain Lakes, NJ) and sealing the capillaries using nail polish. Two capillaries were imaged simultaneously. Signal
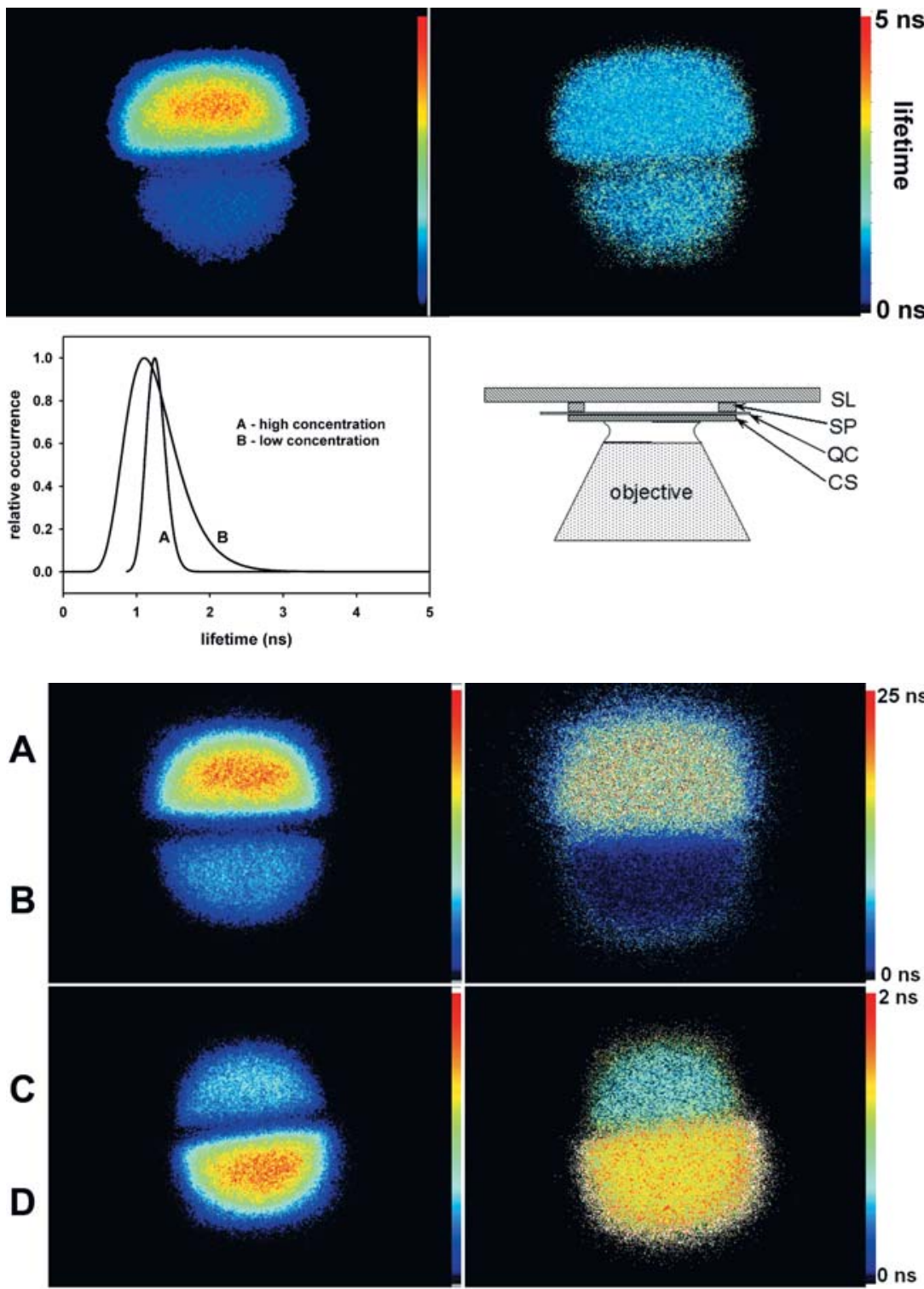

intensity lifetime
FIGURE 6 Intensity-independent fluorophore lifetimes recovered by FLIM. Despite a factor of four difference in POPOP concentration and hence signal intensity (upper left), the 3-gate protocol lifetime map (upper right) showed identical mean lifetimes for the two POPOP samples. A histogram of lifetimes fit to a log-normal function (bottom left) showed a broader distribution for the weaker sample, but the two distributions have identical means: $1.27 \mathrm{~ns}$. The setup is shown (bottom right): quartz slide (SL), silicone spacer (SP), quartz capillary (QC), and cover slip (CS). A non-fluorescent immersion oil (Type FF, Cargille Laboratories, Cedar Grove, NJ) was used to optically couple the objective to the coverslip. The images were of POPOP in ethanol solutions in two quartz capillaries. The capillary interface was on the diameter, with the circular region as the illumination area. The intensity images were background subtracted before calculation of lifetime map

FIGURE 7 Lifetime imaging of fluorophores in quartz capillaries. Top, 9-cyanoanthracene (A) and rose bengal (B); bottom, rose bengal (C) and POPOP (D). Lifetime maps calculated using the 3-gate protocol. Mean lifetime values for the various fluorophores are listed in Table 2. See Fig. 6 for sample mounting setup 
strengths were kept to below 400 counts, and checked to ensure there was no photobleaching. Lifetime images were calculated from three intensity images at 337.1-nm excitation which were separated by $1 \mathrm{~ns}$ increments starting at least $1 \mathrm{~ns}$ after the excitation pulse peak (subsequently called the 3-gate protocol). The ICCD gate width was set to a nominal value of 200 ps.

One property required of fluorescence lifetime imaging was the ability to accurately measure identical lifetimes for samples with different fluorophore concentrations, and hence difference emission intensities. To evaluate this, capillaries containing POPOP in ethanol at $1 / 100$ and $1 / 400$ saturation were imaged simultaneously. Figure 6 shows intensity and lifetime images, along with a histogram of lifetimes for each region. The histogram for the low concentration region has a broader distribution due to the more significant effects of noise. However, the mean of both distributions revealed the same lifetime of $1.27 \mathrm{~ns}$ (within $10 \%$ of the value of $1.40 \mathrm{~ns}$ measured using the FLS), demonstrating and verifying that FLIM lifetimes were intensity independent.

Figure 7 shows intensity and lifetime maps for several of the fluorophores listed in Table 2. Lifetimes determined from the 3-gate protocol showed excellent agreement with FLS measured values and FLIM data obtained from single exponential fits (Fig. 5). Differences compared to FLS lifetimes increased for the longest (9-cyanoanthracene) and shortest (rose bengal) lifetime fluorophores. Differences increased for long lifetimes because the lifetimes determined from three gates in 1-ns intervals did not sufficiently sample the intensity decay. As the fluorophore lifetime decreases, the effects of not deconvolving the system response from the FLIM measurements became significant. The ICCD gate width measured in Sect. 3.3 was $0.62 \mathrm{~ns}$ for the nominal $200 \mathrm{ps}$ setting, and significant differences (21\%) were seen for fluorophores with lifetimes comparable to the gate width.

\section{4}

\section{Simulations of short lifetimes}

Next, we use simulations to quantify the effects of not deconvolving the FLIM system response from the measured decay on the FLIM extracted lifetimes. Temporal decays were simulated by numerically convolving a Gaussian excitation with a single-exponential decay of known lifetime. Apparent lifetimes extracted from the convolution were compared with results from FLIM measurements. In the simulations, the excitation was a Gaussian with a FWHM of $1.14 \mathrm{~ns}$, the width of the system response measured using the ICCD at the sample (Fig. 2a). Apparent lifetimes from the simulations were determined in the same manner as lifetimes extracted using the FLIM, by fitting a single exponential to the decay starting at $0.5 \mathrm{~ns}$ after the simulation peak.

Figure 8 shows the lifetime extracted from the simulations as a function of input lifetime. Extracted lifetimes approach a finite value as input lifetimes approach zero, as expected, representing a single-exponential fit to the tail of the system response. Recovered lifetime differences fall to $5.5 \%$ for a $0.8 \mathrm{~ns}$ input lifetime, comparable to measured error levels in Table 2. Applying the simulation correction to the fastest fluorophore in Table 2, rose bengal in ethanol, an extracted lifetime of $0.70 \mathrm{~ns}$ corresponds to an input lifetime of $0.64 \mathrm{~ns}$,

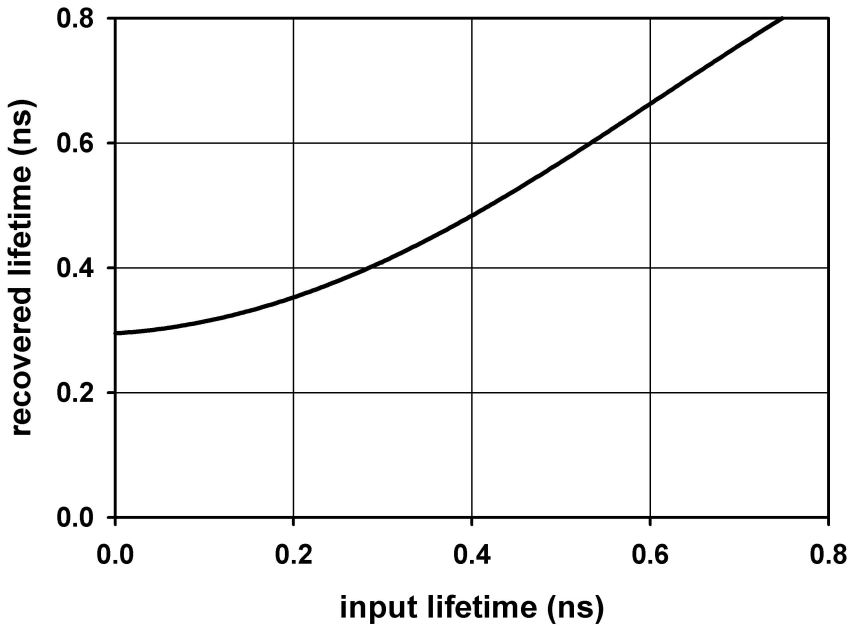

FIGURE 8 Simulation of FLIM recovered lifetime. A Gaussian with a FWHM equal to the measured system response of the system was numerically convolved with a single exponential of known lifetime. The lifetime recovered using the same fitting protocol as Fig. 5 is plotted versus simulation input lifetime. This plot was used to correct experimentally recovered lifetimes of fast fluorophores

reducing the error of the FLIM measurement as compared with the FLS measured lifetime ( $0.62 \mathrm{~ns}$ ) from 13 to $3 \%$.

The simulation was applied to FLIM measurements of a biological fluorophore nicotinamide adenine dinucleotide (NADH) $\left(2 \times 10^{-4} \mathrm{M}\right.$ in phosphate buffered saline(PBS) $)$, which had an FLS measured mean lifetime of $0.36 \mathrm{~ns}$. An exponential fit to the FLIM decay yielded a value of $0.57 \mathrm{~ns}$, corresponding to a lifetime value of $0.50 \mathrm{~ns}$ after applying the simulated correction. The inability to simulate the NADH fluorescence more accurately may be due to deviations in the real system response from the assumed Gaussian pulse shape and to the known multi-exponential behavior of NADH fluorescence [3]. Two measurements each of rose bengal in ethanol and NADH in PBS resulted in a reproducibility of $0.04 \mathrm{~ns}$. Thus the FLIM system was able to discriminate between two fluorophores each with lifetimes significantly shorter than the system response.

\subsection{Temporal discrimination and spatial resolution}

In order to assess the ability of the FLIM system to discriminate between lifetime differences smaller than 100 ps, fluorescence lifetimes were determined from solutions of POPOP at different viscosities, produced by varying the ratio of glycerol to ethanol in the mixture [28]. Lifetimes were determined using the three methods described previously (FLIM exponential fit, FLIM 3-gate protocol, and FLS measured). Between the $80 \%$ - and $60 \%$-ethanol samples, there was an average increase of $0.05 \pm 0.03 \mathrm{~ns}$ detected in the fluorescence lifetime, where the error was the standard deviation amongst the three methods. The reproducibilities of the FLS measured lifetimes for the $80 \%$ - and $60 \%$-ethanol samples were 0.009 and $0.005 \mathrm{~ns}$, indicating that lifetime differences greater than $0.014 \mathrm{~ns}$ were significant. Thus the measured difference of $0.05 \pm 0.03$ ns between the $80 \%$ - and $60 \%$-ethanol samples was statistically significant, and the FLIM system was able to measure lifetime differences at least as small as $50 \mathrm{ps}$. 
The spatial resolution for the $40 \times$ objective was determined using 1- $\mu \mathrm{m}$ Fluoresbrite YG microspheres (17154-1, Polysciences, Inc., Warrington, PA) in de-ionized water placed between a quartz slide and \#1 glass coverslip held in place using a silicone spacer. Fluorescence intensity images were taken at a delay near the peak intensity and analyzed without image averaging. Profile peaks were determined by fits to Gaussians and separations as small as 2.7 pixels could be resolved.

The pixel's spatial size was determined by imaging, using brightfield illumination, similarly mounted $10-\mu \mathrm{m}$ polystyrene microspheres (17136-5, Polysciences, Inc.). After fitting profiles to Gaussians, the center-to-center separation for two beads in contact was $17.43 \pm 0.30$ pixels, where the error was the standard deviation over four measurements. The bead diameter was $9.14 \pm 0.71 \mu \mathrm{m}$, as specified by the manufacturer. Therefore, an image's pixel resolution was $0.52 \pm$ $0.05 \mu \mathrm{m} /$ pixel. Thus the 2.7 pixel resolution described above corresponded to a $1.4-\mu \mathrm{m}$ spatial resolution.

\section{5}

\section{Applications}

The FLIM system detailed above has large spectral tunability and temporal dynamic range, and examples of timeresolved imaging of fluorescence from living biological systems using both these features are presented here. The section highlights applications in metabolic and functional imaging of endogenous biological fluorescence, and oxygen sensing using an exogenous fluorophore on systems of biomedical interest.

The human bronchial epithelial (HBE) cell line BEAS-2B used here has been previously described $[29,30]$, and provided a valuable model for studies seeking to distinguish between normal and malignant cells [31]. BEAS-2B was derived from normal human bronchial epithelial cells, immortalized via transduction of the SV40 T antigen [32]. HBE cells were plated in LHC-9 culture medium (Biofluids, Rockville, MD) on glass-bottomed culture dishes for microscopic imaging (MatTek Corp., Ashland, MA) coated with bovine serum albumin, collagen, and human fibronectin and grown at $37^{\circ} \mathrm{C}$ with $5 \% \mathrm{CO}_{2}$ in a humidified incubator as described in [29]. Cells were used 2-3 days after plating and were imaged within $30 \mathrm{~min}$ at room temperature.

Figure 9 shows representative endogenous fluorescence intensity and lifetime images of living BEAS-2B cells in monolayer. Excitation was at $337.1 \mathrm{~nm}$, and images were col- lected though a 400-600-nm band-pass filter. Lifetime maps were generated using the 3-gate protocol. ICCD gate width was set to the shortest setting. Mean lifetimes were determined from lifetime histograms of a ROI. A mean lifetime of $1.29 \pm 0.09 \mathrm{~ns}$ was determined from five independently cultured dishes, with the uncertainty being the standard deviation. Endogenous fluorescence spectra obtained from HBE cells in suspension (trypsinized, then resuspended in PBS) measured using the FLS were consistent with the spectrum of endogenous $\mathrm{NAD}(\mathrm{P}) \mathrm{H}[33]$.

The FLIM system is compatible with extended functional imaging studies on living cells, thereby allowing us to identify endogenous cellular fluorescence as originating from mitochondrial NADH. Figure 10 shows that the modulation of endogenous fluorescence intensity upon modification of mitochondrial function in living cells is consistent with the action of either a mitochondrial inhibitor (top) or uncoupler (bottom). Cyanide $\left(\mathrm{CN}^{-}\right)$binds to and blocks cytochrome $\mathrm{c}$ oxidase (complex IV) of the electron transport chain in mitochondria, thereby inhibiting oxygen consumption. This drives mitochondrial $\mathrm{NAD}^{+}$into reduction, thus increasing NADH fluorescence intensities [34], as observed in Fig. 10 (top), 10 min after the addition of KCN to a final concentration of $4 \mathrm{mM}$. Carbonyl cyanide 4-(trifluoromethoxy) phenylhydrazone (FCCP) uncouples the electron transport chain from ATP production by increasing proton permeability of the inner mitochondrial membrane. The resulting increased oxygen consumption drives mitochondrial NADH into oxidation, thereby decreasing NADH fluorescence intensities [34], as observed in Fig. 10 (bottom), 9 min after the addition of FCCP to a final concentration of $1 \mu \mathrm{M}$.

FLIM of oxygen-quenched ruthenium-based dyes is emerging as a method for intracellular oxygen sensing [12, 35]. As a demonstration of this method, we stained BEAS-2B cells in monolayer with the oxygen-quenched dye tris $\left(2,2^{\prime}\right.$ bipyridyl)dichloro-ruthenium(II) hexahydrate (RTDP) at a final concentration of $0.3 \mathrm{mM}$ for 4-6 hours prior to imaging. The cells were then washed with PBS at least three times before $1 \mathrm{~mL}$ of PBS or medium was added. Figure 11 shows representative exogenous fluorescence intensity and lifetime images of BEAS-2B cells in monolayer. Excitation was at $460 \mathrm{~nm}$ using the output of the nitrogen-pumped dye laser. Because RTDP has a lifetime two orders of magnitude longer than that of NADH, the 3-gate protocol was replaced by a protocol using four gate delays incremented by $200 \mathrm{~ns}$, starting $200 \mathrm{~ns}$ after the fluorescence emission peak. Additionally,

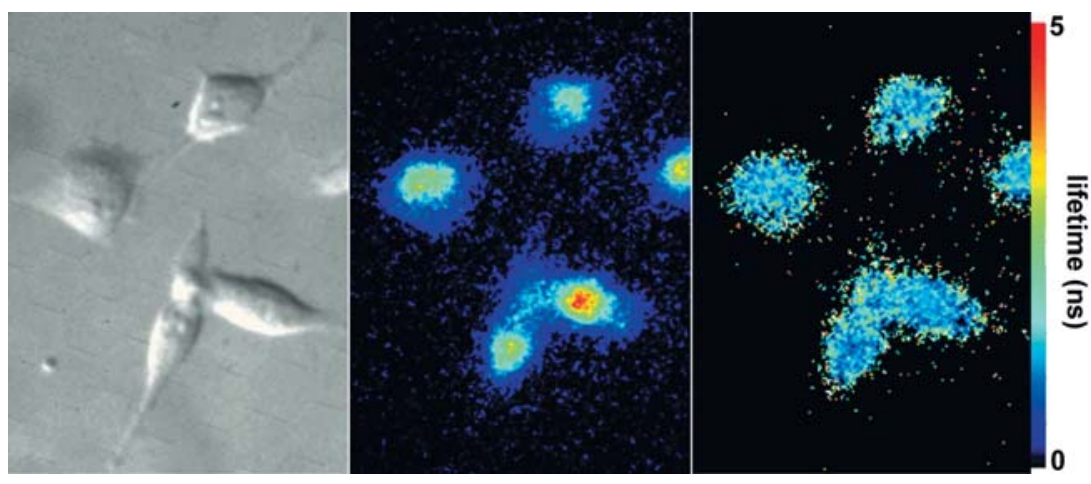

FIGURE 9 Fluorescence lifetime images of endogenous fluorescence from living human cells. Left: Differential interference contrast (DIC) image of BEAS-2B HBE cells. Middle: Endogenous fluorescence intensity image. Right: Fluorescence lifetime image. The mean lifetime was $1.29 \pm 0.09 \mathrm{~ns}$, where the uncertainty was the cell-to-cell standard deviation in five independently grown cell cultures. Lifetimes were determined using the 3-gate protocol 

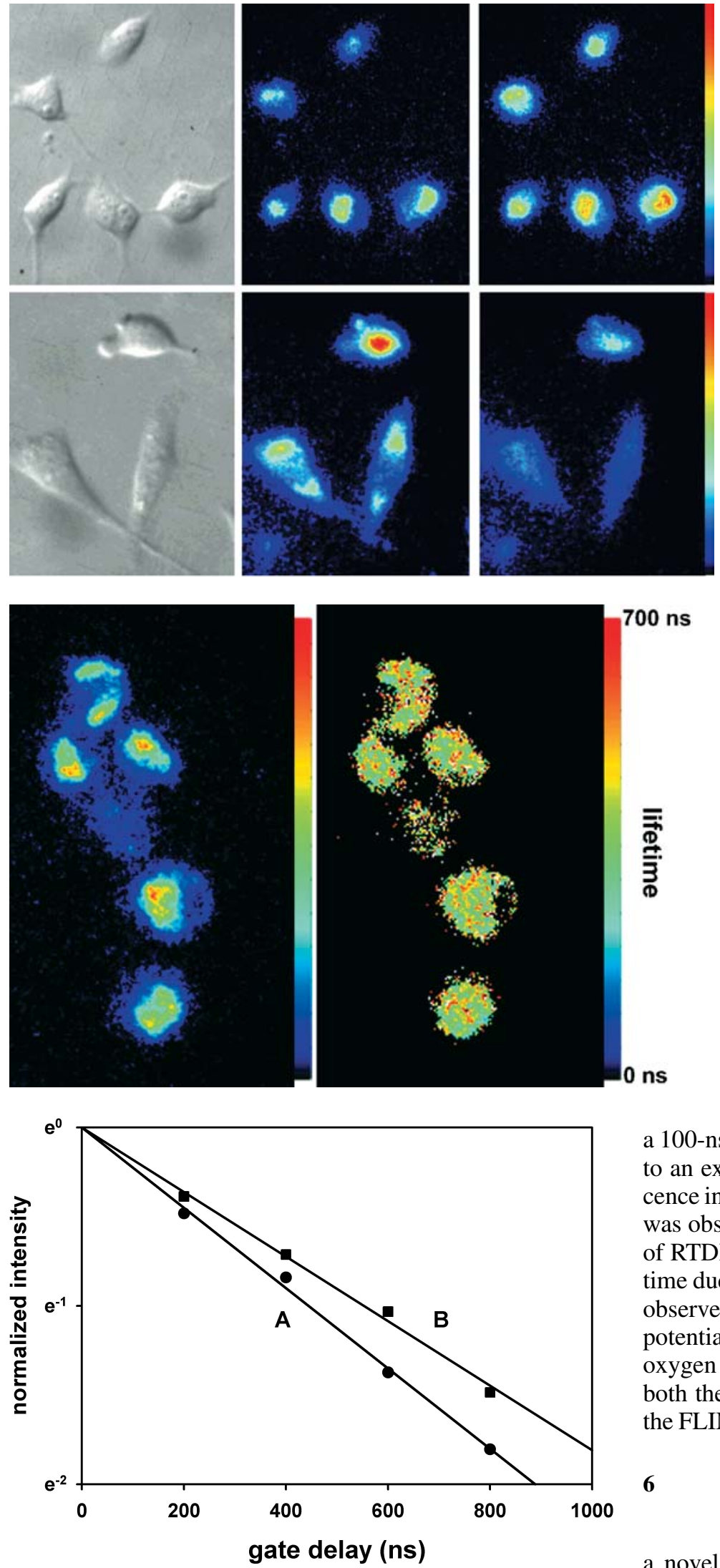

FIGURE 12 A change in mean fluorescence lifetime of intracellular RTDP was detected under air-saturated (A) and oxygen-depleted (B) conditions. The mean fluorescence lifetime of the oxygen-quenched dye RTDP increased by $\sim 100 \mathrm{~ns}$ under oxygen-depleted conditions, produced by flowing nitrogen gas over the sample

\section{6}

FIGURE 10 Functional imaging of living human cells using mitochondrial blockers (top) and uncouplers (bottom). Top (middle, right): fluorescence intensity before and 10 minutes after the addition of $4 \mathrm{mM} \mathrm{KCN}$. Bottom (middle, right): fluorescence intensity before and $9 \mathrm{~min}$ after the addition of $1-\mu \mathrm{M}$ FCCP
FIGURE 11 FLIM oxygen sensing in living cells using the oxygen-quenched dye RTDP. Left: RTDP fluorescence intensity. Right: fluorescence lifetime. The excitation was at $460 \mathrm{~nm}$. The mean fluorescence lifetime was $407 \mathrm{~ns}$

a 100-ns ICCD gate width, achieved by slaving the intensifier to an external logic pulse, was used to capture each fluorescence intensity image. A mean fluorescence lifetime of $407 \mathrm{~ns}$ was observed, consistent with previously measured lifetimes of RTDP [12]. An increase in mean RTDP fluorescence lifetime due to a decrease in dissolved oxygen concentration was observed upon flowing nitrogen gas over the sample (Fig. 12), potentially making the FLIM system useful for intracellular oxygen sensing in living cells. This application demonstrates both the tunability and the large temporal dynamic range of the FLIM system.

\section{Discussion}

We report the design and characterization of a novel, tunable fluorescence lifetime imaging microscope system for laser-based biological sensing with picosecond resolution operating in the UV-visible-NIR region of the electromagnetic spectrum. The system (Fig. 1a) was capable of spatially resolving electronic excited state lifetimes from 
living biological samples under investigation, thus revealing information on local biomolecular environment.

The system operated in the wide-field with time-resolved images captured from the lateral port of an inverted microscope using a gated intensified CCD camera, with a minimum nominal gate width of $200 \mathrm{ps}$. The system used a pulsed nitrogen-pumped dye laser with output from the UV to NIR. Fluorescence lifetimes were determined in the time domain by fitting a decay to a series of time-gated intensity images on a pixel-by-pixel basis using (13). Typical acquisition and analysis times for lifetime images were 10-20 s.

The FLIM system was tested and calibrated for accurate data collection and lifetime determination using fluorescence lifetime standards for reference (Table 2; Figs. 5 and 7). The ICCD's intensity response was characterized to ensure stability and linearity (Fig. 4) for accurate sampling of the fluorescence decay intensity. As noted previously [36,37], effects due to non-uniform illumination are important in fluorescence intensity imaging. These effects should not distort the intensity-independent lifetime image [38]. The ability to recover identical lifetimes from samples of dissimilar fluorescence intensities was verified experimentally (Fig. 6).

The system response (Fig. 3) for the various components of the FLIM system (the total system response at the sample (Fig. 2), the effective ICCD gate width, and the laser pulse duration) was determined in order to gauge the temporal resolution of the system. Without system response deconvolution, accurate lifetimes could be determined to about one-half of the system response, or $0.6 \mathrm{~ns}$.

The de facto method for removing the system response from time-resolved fluorescence decay is iterative reconvolution in which a simulation of a fluorescence signal - an exponential decay convolved with the measured system response - is iteratively performed until the difference between simulation and measured fluorescence decay is minimized. Such a procedure is not routinely practical for imaging applications because the iterative reconvolution algorithm can be computationally intensive when performed on a pixel-bypixel basis. In addition, the need for increased sampling of the fluorescence response of the sample would increase data acquisition times and could lead to sample photodamage.

Simulations (Sect. 4.4 and Fig. 8) showed that taking into account the system response improved lifetime determinations below $0.6 \mathrm{~ns}$. Thus iterative reconvolution may still be desirable for improved accuracy in lifetime determination, in which case, only a small region of interest (perhaps a part of the cell) would be analyzed to decrease computation time. Experience with a non-imaging fluorescence lifetime spectrometer system [21] has shown that sampling increments of $0.25 \mathrm{~ns}$ provide sufficient sampling for lifetime determination to $\sim 0.4 \mathrm{~ns}$, or one-tenth of the system response ( $4 \mathrm{~ns}$ in that report).

The ability of the FLIM system to excite into the UV makes several endogenous fluorophores important in biomedicine $[15,39]$ accessible for fluorescence lifetime study. These include metabolic co-factors such as reduced nicotinamide adenine dinucleotide (NADH) and structural proteins such as collagen and elastins. NADH excites in the near$\mathrm{UV}(\sim 340 \mathrm{~nm})$ and emits at $\sim 460 \mathrm{~nm}$. (The abbreviation $\mathrm{NAD}(\mathrm{P}) \mathrm{H}$ is often used to emphasize the spectral indistin- guishability of NADH and NADPH in cellular systems.) $\mathrm{NAD}(\mathrm{P}) \mathrm{H}$ fluorescence intensity has been used to monitor metabolism using ratiometric methods [40] and to infer oxygen consumption rates using mitochondrial-function modifiers [34]. Here, we showed FLIM imaging of endogenous NADH in living cells imaged for several minutes, thereby allowing functional imaging studies to be performed (Figs. 9 and 10). Structural proteins collagen and elastin are major fluorophores found in biological tissues. Fluorescence lifetime methods may be useful in tissue studies because biochemical and morphological factors such as cellular metabolism, vascularization, mucosa thickness, and cell density affect measured fluorescence decays [41,42]. Lifetime spectroscopy in tissues involving endogenous fluorescence has been used in the detection of early cancers and staging of atherosclerotic plaques [19,43-48].

Fluorescence lifetime imaging of near UV fluorophores is most often performed using non-linear excitation processes, such as two-photon absorption $[16,17,49]$. Because the excitation light must be tightly focused onto a sample in order to offset effects of small non-linear excitation cross sections, raster-scanning is used to form an image. Out of focal plane excitation is eliminated and fluorescence is emitted only from the small focal volume, providing axial sectioning analogous to confocal imaging [18]. A common excitation source in twophoton systems is the Ti:Sapphire laser, useful for its ultrashort pulses $(\sim 100 \mathrm{fs})$, high repetition rate $(\sim 100 \mathrm{MHz})$, exceptional stability, and tunability, with a typical spectral range of 700-1000 $\mathrm{nm}$ for two-photon excitation (corresponding to a one-photon excitation range of $350-500 \mathrm{~nm}$ ).

Direct UV excitation of endogenous fluorophores with a nitrogen laser source provides a significantly less expensive, wide-field, and potentially portable alternative to multiphoton excitation for sub-nanosecond FLIM imaging of biological samples. A nitrogen source emits at $337.1 \mathrm{~nm}$, a wavelength commonly used in clinical endogenous fluorescence studies [15]. Non-imaging time-resolved clinical spectrofluorimeters based on nitrogen lasers have been reported [19-21], making comparisons between in vitro imaging and in vivo non-imaging studies possible. The choice of FLIM implementation depends on the specific application, availability of experimental equipment, and the nature of the lifetime information to be extracted.

Because the FLIM system presented here uses a pulsed dye laser, the excitation wavelength is tunable from the UV through NIR (337-960 nm) part of the electromagnetic spectrum. Wavelengths were easily changed (few seconds) by means of "flippable" mirrors that either directed the nitrogen laser output to the microscope for direct UV excitation at $337.1 \mathrm{~nm}$, or into the dye laser for wavelengths dependent on the dye used. Fluorescence lifetime imaging applications at 337.1 and $460 \mathrm{~nm}$ excitation were presented demonstrating the tunability and large temporal dynamic range of the system. The applications included the lifetime imaging of the endogenous metabolic cofactor NADH (Fig. 9) and an exogenous oxygen-quenched ruthenium dye (Fig. 11) which utilized the 337.1 and $460 \mathrm{~nm}$ excitation wavelengths, respectively. Optics (Fig. 1a) can be aligned so that re-tuning is not necessary after changing wavelengths, making possible nearsimultaneous imaging of several fluorophores in living cells. 
ACKNOWLEDGEMENTS This work was supported by The Whitaker Foundation (M.-A.M. and E.D.), the Andrew W. Mellon Foundation (M.-A.M.), Dartmouth College's Burke Research Initiation Award (M.-A.M.), the National Institutes of Health and National Cancer Institute Grant No. RO-1 CA87546 (E.D.), a Samuel Waxman Foundation Cancer Research Award (E.D.), and the Oracle Giving Fund (E.D.). J.A.B. and F.K.M. were supported by an award to Dartmouth College from the National Science Foundation's Research Experience for Undergraduates Program DMR9820408. We wish to thank E.A. Hamon, K. Heyman, and M. Bussey for technical assistance.

\section{REFERENCES}

1 W. Rudolph, M. Kempe: J. Mod. Opt. 44, 1617 (1997)

2 W.T. Mason: Fluorescent and Luminescent Probes for Biological Activity (Academic Press, London 1999)

3 J.R. Lakowicz: Principles of Fluorescence Spectroscopy (Kluwer Academic/Plenum, New York 1999)

4 P.J. Tadrous: J. Pathol. 191, 229 (2000)

5 I. Bugiel, K. König, H. Wabnitz: Lasers Life Sci. 3, 47 (1989)

6 X.F. Wang, A. Periasamy, B. Herman, D. Coleman: Crit. Rev. Anal. Chem. 23, 369 (1992)

7 T. French, P.T.C. So, C.Y. Dong, K.M. Berland, E. Gratton: Methods Cell Biol. 56, 277 (1998)

8 T.W.J. Gadella, Jr.: 'Fluorescence Lifetime Imaging Microscopy (FLIM): Instrumentation and Application'. In Fluorescent and Luminescent Probes for Biological Activity, ed. by W.T. Mason (Academic Press, San Diego 1999)

9 R. Cubeddu, D. Comelli, C. D’Andrea, P. Taroni, G. Valentini: J. Phys. D: Appl. Phys. 35, R61 (2002)

10 P.K. Urayama, M.-A. Mycek: Fluorescence Lifetime Imaging Microscopy of Endogenous Biological Fluorescence. In Handbook of Biomedical Fluorescence, ed. by M.-A. Mycek, B.W. Pogue (MarcelDekker, New York 2003) pp. 211-236

11 R. Sanders, A. Draaijer, H.C. Gerritsen, P.M. Houpt, Y.K. Levine: Anal. Biochem. 227, 302 (1995)

12 H.C. Gerritsen, R. Sanders, A. Draaijer, Y.K. Levine: J. Fluoresc. 7, 11 (1997)

13 J.R. Lakowicz, H. Szmacinski, K. Nowaczyk, M.L. Johnson: Proc. Natl. Acad. Sci. 89, 1271 (1992)

14 T. Oida, Y. Sako, A. Kusumi: Biophys. J. 64, 676 (1993)

15 N. Ramanujam: Neoplasia 2, 89 (2000)

16 D.W. Piston, D.R. Sandison, W.W. Webb: Proc. SPIE 1640, 379 (1992)

17 P.T.C. So, T. French, W.M. Yu, K.M. Berland, C.Y. Dong, E. Gratton: Bioimaging 3, 49 (1995)

18 P.T.C. So, C.Y. Dong, B.R. Masters, K.M. Berland: Annu. Rev Biomed. Eng. 2, 399 (2000)

19 M.-A. Mycek, K. Schomacker, N. Nishioka: Gastrointest. Endosc. 48 390 (1998)

20 T. Glanzmann, J.-P. Ballini, H. van den Bergh, G. Wagnieres: Rev. Sci. Instrum. 70, 4067 (1999)

21 J.D. Pitts, M.-A. Mycek: Rev. Sci. Instrum. 72, 3061 (2001)
22 X.F. Wang, T. Uchida, D.M. Coleman, S. Minami: Appl. Spectrosc. 45, 360 (1991)

23 A. Periasamy, P. Wodnicki, X.F. Wang, S. Kwon, G.W. Gordon, B. Herman: Rev. Sci. Instrum. 67, 3722 (1996)

24 M.J. Cole, J. Siegel, S.E.D. Webb, R. Jones, K. Dowling, M.J. Dayel, D. Parsons-Karavassilis, P.M.W. French, M.J. Lever, L.O.D. Sucharov, M.A.A. Neil, R. Juskaitis, T. Wilson: J. Microsc. 203, 246 (2001)

25 S.E.D. Webb, Y. Gu, S. Leveque-Fort, J. Siegel, M.J. Cole, K. Dowling, R. Jones, P.M.W. French, M.A.A. Neil, R. Juskaitis, L.O.D. Sucharov, T. Wilson, M.J. Lever: Rev. Sci. Instrum. 73, 1898 (2002)

26 W.R. Ware, M. Pratinidhi, R. Bauer: Rev. Sci. Instrum. 54, 1148 (1983)

27 K.K. Sharman, A. Periasamy, H. Ashworth, J.N. Demas, N.H. Snow: Anal. Chem. 71, 947 (1999)

28 M.-A. Mycek, P.K. Urayama, K. Heyman, M. Bussey: Proc. SPIE Int. Symp. Biomed. Opt. (2003)

29 J. Langenfeld, F. Lonardo, H. Kiyokawa, T. Passalaris, M. Ahn, A. Rusch, E. Dmitrovsky: Oncogene 13, 1983 (1996)

30 J. Pitts, R. Sloboda, K. Dragnev, E. Dmitrovsky, M.-A. Mycek: J. Biomed. Opt. 6, 31 (2001)

31 K.H. Dragnev, J.R. Rigas, E. Dmitrovsky: Oncol. 5, 361 (2000)

32 R. Reddel, Y.K. Ke, V. Gerwin, M. McMenamin, J. Lechner, R. Su, D. Brash, J.-B. Park, J. Limb Rhim, C. Harris: Cancer Res. 48, 1904 (1988)

33 M.-A. Mycek, J. Pitts, R. Sloboda, K. Dragnev, E. Dmitrovsky: Biophys. J. 80, 365a (2001)

34 M.R. Duchen, T.J. Biscoe: J. Physiol. 450, 13 (1992)

35 F.N. Castellano, J. Lakowicz, R: Photochem. Photobiol. 67, 179 (1998)

36 T.D. Wang, J. Van-Dam, J.M. Crawford, E.A. Preisinger, Y. Wang, M.S. Feld: Gastroenterol. 111, 1182 (1996)

37 B. Chwirot, S. Chwirot, W. Jedrzejczyk, M. Jackowski, A. Raczynska, J. Winczakiewicz, J. Dobber: Lasers Surgery Med. 21, 149 (1997)

38 J. Lakowicz: Principles of Fluorescence Spectroscopy (Plenum Press, New York 1983)

39 R. Richards-Kortum, E. Sevick-Muraca: Annu. Rev. Phys. Chem. 47, 555 (1996)

40 B. Chance, B. Schoener, R. Oshino, F. Itshak, Y. Nakase: J. Biol. Chem. 254, 4764 (1979)

41 K. Vishwanath, B.W. Pogue, M.-A. Mycek: Phys. Med. Biol. 47, 3387 (2002)

42 M.-A. Mycek, K. Vishwanath, B.W. Pogue, K.T. Schomacker, N.S. Nishioka: Proc. SPIE Int. Symp. Biomed. Opt. (2003)

43 A. Pradhan, B.B. Das, K.M. Yoo, J. Cleary, R. Prudente, E. Celmer, R.R. Alfano: Lasers Life Sci. 4, 225 (1992)

44 L. Pfeifer, K. Schmalzigaug, R. Paul, J. Lichey, K. Kemnitz, F. Fink: Proc. SPIE 2627, 129 (1995)

45 B.B. Das, L. Feng, R.R. Alfano: Rep. Prog. Phys. 60, 227 (1997)

46 B. Beauvoit, B. Chance: Mol. Cell. Biochem. 184, 445 (1998)

47 J. Mizeret, T. Stepinac, M. Hansroul, A. Studzinski, H. van den Berg, G. Wagnieres: Rev. Sci. Instrum. 70, 4689 (1999)

48 M.-A. Mycek, K. Vishwanath, K.T. Schomacker, N.S. Nishioka: 'Fluorescence Spectroscopy for In Vivo Discrimination of Pre-Malignant Colonic Lesions'. In Biomedical Topical Meetings, Optical Society of America Technical Digest (2000)

49 J. Sytsma, J.M. Vroom, C.J. De Grauw, H.C. Gerritsen: J. Microsc. 191, 39 (1998) 